\title{
The RECOVER I: A multicenter prospective study of Impella 5.0/LD for postcardiotomy circulatory support
}

\author{
Bartley P. Griffith, MD, ${ }^{\mathrm{a}}$ Mark B. Anderson, MD, ${ }^{\mathrm{b}}$ Louis E. Samuels, MD, ${ }^{\mathrm{c}}$ Walter E. Pae, Jr, MD, ${ }^{\mathrm{d}}$ \\ Yoshifumi Naka, MD, PhD, ${ }^{e}$ and O. Howard Frazier, $\mathrm{MD}^{\mathrm{f}}$
}

\begin{abstract}
Objectives: Cardiogenic shock after cardiac surgery is accompanied by a high mortality rate. Early institution of hemodynamic support with a versatile, easy to insert left ventricular assist device might help bridge patients to recovery or to the next therapy, and improve the outcomes.
\end{abstract}

\begin{abstract}
Methods: Patients developing cardiogenic shock or low cardiac output syndrome after being weaned off cardiopulmonary bypass were enrolled in a prospective single-arm feasibility study (RECOVER I). The primary safety endpoint was the frequency of major adverse events (death, stroke) at 30 days or discharge, whichever was longer. The primary efficacy endpoint was survival of the patient to implementation of the next therapy, which included recovery at 30 days after device removal and bridge-to-other-therapy.
\end{abstract}

Results: Sixteen patients provided informed consent and were enrolled in the study. Hemodynamics improved immediately after the initiation of mechanical support: cardiac index, 1.65 versus $2.7 \mathrm{~L} / \mathrm{min} / \mathrm{m}^{2}(P=.0001)$; mean arterial pressure, 71.4 versus $83.1 \mathrm{~mm} \mathrm{Hg}(P=.01)$; and pulmonary artery diastolic pressure, 28.0 versus $19.8 \mathrm{~mm} \mathrm{Hg}(P<.0001)$. The pump provided an average of $4.0 \pm 0.6 \mathrm{~L} / \mathrm{min}$ of flow for an average duration of 3.7 \pm 2.9 days (range, $1.7-12.6)$. The primary safety endpoint occurred in 2 patients $(13 \% ; 1$ stroke and 1 death). For the primary efficacy endpoint, recovery of the native heart function was obtained in $93 \%$ of the patients discharged, with bridge-to-other-therapy in 7\%. Survival to 30 days, 3 months, and 1 year was $94 \%, 81 \%$, and $75 \%$, respectively.

Conclusions: The use of the Impella 5.0/left direct device is safe and feasible in patients presenting with postcardiotomy cardiogenic shock. The device was rapidly inserted, enabled early support, and yielded favorable outcomes. (J Thorac Cardiovasc Surg 2013;145:548-54)

Patients with postcardiotomy cardiogenic shock (PCCS) continue to have poor outcomes. Despite recent advances in surgical techniques and aggressive therapy with increased inotropic support and counterpulsation intra-aortic balloon pumps (IABPs), the overall mortality is frustratingly high. ${ }^{1,2}$ For those patients refractory to inotropic and/or IABP therapy, studies have shown that early ventricular assist device (VAD) implantation improves survival. $^{3-5}$ However, the use of current surgical VADs for

From the Department of Cardiac Surgery, ${ }^{\text {a }}$ University of Maryland Baltimore, Baltimore, Md; Department of Cardiothoracic Surgery, ${ }^{\mathrm{b}}$ Robert Wood Johnson University Hospital, New Brunswick, NJ; Department of Cardiothoracic Surgery, ${ }^{c}$ Lankenau Medical Science Hospital, Wynnewood, Pa; Department of Cardiothoracic Surgery, ${ }^{\mathrm{d}}$ Penn State Hershey Heart and Vascular Institute, Penn State Milton S. Hershey Medical Center, Hershey, Pa; Department of Surgery, ${ }^{\mathrm{e}}$ New York-Presbyterian Hospital/Columbia Milstein Hospital, New York, NY; and Department of Cardiothoracic Surgery, ${ }^{\mathrm{f}}$ Tex Heart Institute, St. Luke's Episcopal Hospital, Houston, Tex.

Food and Drug Administration Investigational Device Exemption (IDE No. G030202) was obtained by the study sponsor (Abiomed, Danvers, Mass).

Disclosures: Authors have nothing to disclose with regard to commercial support.

Received for publication Nov 18, 2011; revisions received Dec 30, 2011; accepted for publication Jan 24, 2012; available ahead of print March 12, 2012.

Address for reprints: Bartley P. Griffith, MD, Department of Cardiac Surgery, University of Maryland Baltimore, 22 South Greene Street, N4W94, Baltimore, MD 21201 (E-mail: BGRIFFITH@smail.umaryland.edu).

$0022-5223 / \$ 36.00$

Copyright @ 2013 Published by Elsevier Inc. on behalf of The American Association for Thoracic Surgery

doi:10.1016/j.jtcvs.2012.01.067
PCCS remains limited, because they require significant incremental surgery and highly trained operative and postoperative teams. Moreover, no guidelines have been established for patient selection for the early use of VADs in this setting.

The Impella 5.0/left direct (LD) (Abiomed, Danvers, MA) is a minimally invasive, $21 \mathrm{~F}$, rotary blood pump mounted on a 9F driveline/bearing purge delivery catheter (Figure 1). The pump is positioned retrogradely in the left ventricle (LV) across the aortic valve. When in function, the pump aspirates the blood from the LV and expels it into the ascending aorta, providing up to $5 \mathrm{~L} / \mathrm{min}$ of forward flow into the general circulation. The pump actively unloads the LV and increases the coronary perfusion, thereby improving the myocardium oxygen supply/demand ratio. ${ }^{6,7}$ Considering its relative ease of insertion and potential beneficial hemodynamic effect, the Impella 5.0/LD might fill a technology gap for perioperative patients who require early and potent circulatory support to restore normal cardiac function or to bridge them to the next therapy.

The RECOVER I study was a prospective, single-arm, clinical trial designed under US Food and Drug Administration guidance to investigate, at a maximum of 7 sites, the safety and feasibility of the Impella 5.0/LD in up to 20 patients experiencing cardiogenic shock (CS) or low cardiac output syndrome after cardiac surgery. 


\section{Abbreviations and Acronyms \\ $\mathrm{CPB}=$ cardiopulmonary bypass \\ $\mathrm{CS}=$ cardiogenic shock \\ IABP $=$ intra-aortic balloon pump \\ $\mathrm{LD}=$ left direct \\ $\mathrm{LV}=$ left ventricle \\ $\mathrm{LVAD}=$ left ventricular assist device \\ PCCS $=$ postcardiotomy cardiogenic shock \\ $\mathrm{VAD}=$ ventricular assist device}

\section{METHODS}

\section{Study Population}

Eligible patients were those with low cardiac output (cardiac index, $\geq 1.3$ but $\leq 2.2 \mathrm{~L} / \mathrm{min} / \mathrm{m}^{2}$ ) and elevated filling pressures (pulmonary capillary wedge pressure, $\geq 20$ but $\leq 30 \mathrm{~mm} \mathrm{Hg}$ or pulmonary artery diastolic pressure $\geq 25$ but $\leq 35 \mathrm{~mm} \mathrm{Hg}$ ) after weaning from cardiopulmonary bypass (CPB) despite stable infusion of 1 high-dose inotrope or 2 mediumdose inotropes (considered medium and high dose for epinephrine $<0.03$ and $\geq 0.03 \mu \mathrm{g} / \mathrm{kg} / \mathrm{min}$, respectively, for $\geq 15$ minutes; for dobutamine and dopamine $<5$ and $\geq 5 \mu \mathrm{g} / \mathrm{kg} / \mathrm{min}$, respectively, for $\geq 15$ minutes; and milrinone $<0.3$ and $\geq 0.3 \mu \mathrm{g} / \mathrm{kg} / \mathrm{min}$, respectively, for $\geq 120$ minutes). The exclusion criteria were age younger than 18 or older than 75 years, elevated blood urea nitrogen of $100 \mathrm{mg} / \mathrm{dL}$ or more, renal failure (creatinine $\geq 3.5 \mathrm{mg} / \mathrm{dL}$ ), hepatic dysfunction (total bilirubin $\geq 5 \mathrm{mg} / \mathrm{dL}$ ), and the presence of any cardiac assist device other than IABP. Additional exclusion criteria were right ventricular failure (defined as central venous pressurepulmonary capillary wedge pressure $\geq 10 \mathrm{~mm} \mathrm{Hg}$ or central venous pressure-pulmonary artery diastolic pressure $\geq 10 \mathrm{~mm} \mathrm{Hg}$ or cardiac ascites), evidence of any vascular disease that would preclude placement of the peripheral device, and evidence of left ventricular or right ventricular thrombus. The remaining exclusion criteria were aortic insufficiency ( $\geq 2+$ echocardiographic), aortic valve stenosis (valve orifice area $\leq 1.5$ $\mathrm{cm}^{2}$ ), the presence of a mechanical aortic valve, obstructive or hypertrophic cardiomyopathy, uncorrected ventricular or atrial septal defect, patent foramen ovale, mechanical manifestation of acute myocardial infarction, disorder causing fragility of blood cells or hemolysis, a cardiopulmonary resuscitative maneuver lasting longer than 15 minutes within 24 hours before pump implantation, active systemic infection, sustained or nonsustained ventricular tachycardia or ventricular fibrillation unresponsive to medical therapy, and participation in another investigational drug or device trial.

\section{Device and Procedures}

The device was inserted through a direct or peripheral approach. When a direct insertion, the device was inserted through a 10 -mm vascular graft sewn to the ascending aorta and advanced across the aortic valve into the LV. When a peripheral insertion, the device was introduced through a femoral artery cut down. Using a guidewire, the device was then advanced retrogradely under fluoroscopy or transesophageal echocardiography guidance across the aortic valve and positioned in the LV. When the chest tube drainage decreased to less than $100 \mathrm{~mL} / \mathrm{hr}$, a continuous intravenous infusion of heparin was recommended to achieve a partial thromboplastin time of 40 to 50 seconds.

Per protocol, the recommended duration of support was up to 7 days. The timing of device removal was left to the discretion of the physician. For patients in whom hemodynamic stability was achieved, weaning was started in a stepwise fashion by decreasing the pump speed. Once the speed was reduced to the minimal level of support and well tolerated for about 2 hours, the patient returned to the operating room for device removal. In the case of direct insertion, the pump was retracted into the vascular graft sleeve that was oversewn and then removed. Peripherally placed devices were retrieved from the femoral insertion site either in the operating room or at the bedside.

\section{Study Procedures}

Transthoracic echocardiography was performed before and after pump insertion, at pump removal, at 30 days or at discharge, and 3 months after the procedure. The echocardiographic studies were analyzed by an independent core laboratory (Duke University Clinical Research Institute Echocardiographic Laboratory). Hemodynamic measurements were recorded before and during support using a Swan-Ganz catheter for continuous cardiac output monitoring. The heart rate and blood pressure were monitored after pump and central catheter removal. Serial blood sampling was obtained before, during, and after support for cardiac enzymes, plasma free hemoglobin, biochemistry, blood gases, and hematologic features. If practical, a neurologic assessment was performed before insertion, daily until hospital discharge, and at 30 days and 3 months.

The institutional review board of each center approved the study. When possible, written informed consent was obtained from each patient preoperatively if the patient was considered to be a high-risk candidate for surgery. Alternatively, written informed consent was obtained from the next of kin during surgery in emergency situations according to the protocol. The Clinical Trial Registration Information unique identifier was NCT00596726.

Study data were collected prospectively by research coordinators on case report forms and verified against source documentation by independent trial monitors. An independent thoracic surgeon with expertise in circulatory support adjudicated all adverse events.

\section{Study Endpoints}

The primary safety endpoint was the frequency of major adverse events, defined as death or stroke at 30 days or discharge, whichever was longer. Device malfunction and other complications were included in the adverse event analysis. The primary efficacy endpoint was survival of the patient to the implementation of the next therapy, which included recovery, defined as 30-day survival after removal or hospital discharge (whichever was longer) with no other mechanical support or IABP, and bridge-to-other-therapy, defined as survival to induction of anesthesia for surgery for cardiac transplantation or placement of an implantable VAD. The secondary efficacy endpoints included improved hemodynamics, device placement and technical success, time-to-recovery and reduction of inotropic/pressor support compared with before device placement.

\section{Statistical Analysis}

The data are expressed as the mean \pm standard deviation, median (range), or proportions, as appropriate. Univariate parametric analysis was performed using a paired Student's $t$ test or analysis of variance with repeated measures test. The Wilcoxon sign rank test was used for pair wise nonparametric comparisons. The probability values were 2 tailed. All investigators had access to study data.

\section{RESULTS \\ Patient Characteristics}

From October 25, 2006, to May 16, 2008, 16 patients provided written informed consent and enrolled at 4 US sites. Thirteen were men and 3 were women, with a mean age of $58.4 \pm 9$ years (range, 43-72), and all were high-risk candidates for cardiac surgery (mean logistic European System for Cardiac Operative Risk Evaluation, $34 \% \pm 26 \%$; range, $10-84 \%$ ). They presented with poor left ventricular function (mean ejection fraction, $23 \% \pm 7 \%$; range, $10-35 \%$ ), and multiple co-morbidities, including heart

\section{The Journal of Thoracic and Cardiovascular Surgery $\cdot$ Volume 145, Number 2}


9 French catheter

21 French micro-axial pump

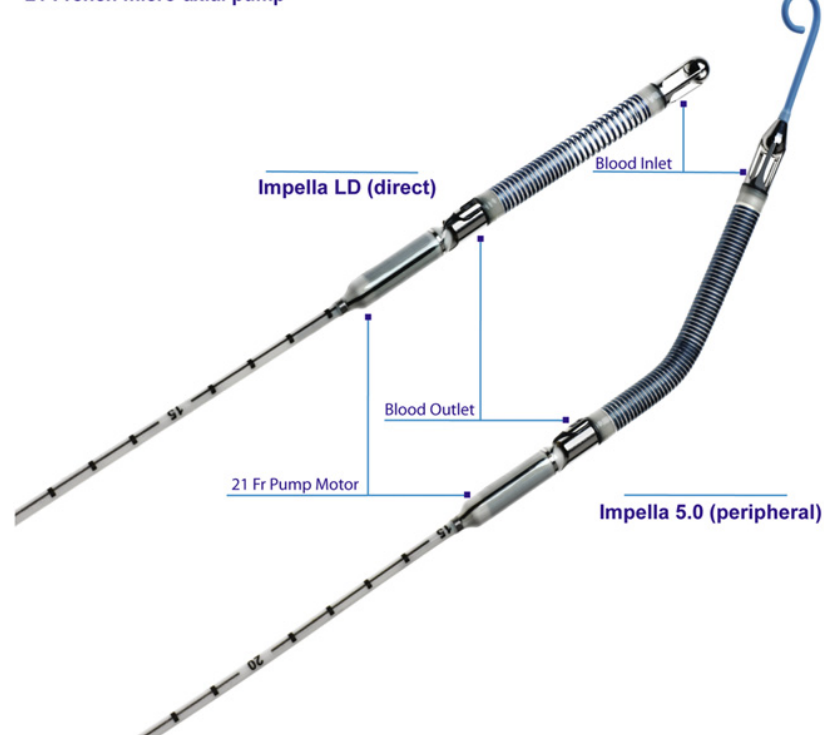

FIGURE 1. Impella 5.0/LD left ventricular assist device (LVAD) (with permission from Abiomed, Danvers, Mass).

failure $(88 \%)$, myocardial infarction $(69 \%)$, unstable angina $(44 \%)$, diabetes mellitus $(38 \%)$, cardiogenic shock $(31 \%)$, and IABP $(25 \%)$. Of the 16 patients, $11(69 \%)$ underwent coronary artery bypass grafting, $3(19 \%)$ underwent combined coronary artery bypass grafting and mitral valve replacement surgery, $1(6 \%)$ underwent mitral valve replacement surgery, and $1(6 \%)$ underwent heart transplant surgery. Of the 16 patients, 11 experienced cardiogenic shock within minutes after weaning off CPB: 2 patients within less than an hour, 2 patients within less than 8 hours, and 1 patient within 24 hours.

\section{Procedural Characteristics}

The Impella device was inserted peripherally in 5 patients $(31 \%)$ and directly in $11(69 \%)$. Device placement was rated as "easy" for $75 \%$ of the cases and "acceptable" for $25 \%$. The time of insertion was less than 5 minutes for 1 patient $(6 \%)$, less than 10 minutes in $6(38 \%)$, longer than 10 but less than 15 minutes in $6(38 \%)$, and longer than 15 minutes in 3 patients $(18 \%)$. The mean duration of circulatory support was $3.7 \pm 2.9$ days (range, 1.7-12.6), and the mean pump flow during support was $4.0 \pm 0.6 \mathrm{~L} /$ $\mathrm{min}$ at the average performance level of 7.7 set by the operator (performance level range, 0-9) and $2.54 \pm 0.9 \mathrm{~L} / \mathrm{min}$ during weaning. There were 2 device malfunctions, most likely due to catheter kinking causing a failure of the purge system for both pumps on days 2 and 3 after insertion. One patient had a replacement device inserted, and the other patient had recovered and was scheduled for device removal the day of the device malfunction. Both patients were alive at the 1-year study visit. Removal of the Impella 5.0/LD was uneventful in all patients.

\section{Safety Endpoint}

The primary safety endpoint (the frequency of major adverse events [death or stroke] at 30 days or discharge, whichever was longer) occurred in 2 patients $(13 \%)$ (1 death and 1 nonfatal stroke). The patient who died experienced early complications during his heart transplant surgery, with profuse bleeding, renal failure, and metabolic acidosis that culminated in CS. Despite hemodynamic stabilization after Impella implantation on postoperative day 1, the patient continued to deteriorate. Support was withdrawn on 3 days after device implantation, and the patient died shortly thereafter. The patient who experienced a nonfatal stroke was admitted in CS on IABP support and mechanical assisted ventilation after failed percutaneous coronary intervention. This patient underwent emergent coronary artery bypass grafting followed by hemodynamic support with the Impella 5.0 left ventricular assist device (LVAD). Shortly afterward, the patient started showing signs of right heart failure and was subsequently supported with a Levitronix right VAD. After removal of the devices, a clinical examination was possible and raised concerns about the patient's neurologic status. A computed tomography scan revealed bilateral multiple cerebral ischemic infarcts. The investigators were unable to determine the time of the onset of the stroke.

One patient had hemolysis for which the relatedness to the device could not be excluded. Six patients developed sepsis. Of those 6 patients, 1 was supported with the device for 12.6 days and the relatedness to the device could not be ruled out (indwelling catheter). Eleven patients had continuous respiratory support for longer than 48 hours after the procedure, 7 patients developed bleeding requiring reoperation ( 3 from the sternal wound, 1 with hemothorax in the context of a coagulopathy, 1 with cardiac tamponade, 1 with a small chest cavity hematoma, and 1 with a remote femoral artery vein patch rupture), 3 patients had renal failure, 1 patient had hepatic failure, and 1 patient had a remote vascular injury (vein patch rupture). No cases of limb ischemia, vascular or cardiac perforation, thrombocytopenia, ventricular fibrillation, or ventricular tachycardia were reported.

There was no echocardiographic evidence of devicerelated injury or dysfunction to the aortic or mitral valve, papillary muscles, chordae, or ventricular cavity during or after device use. At 30 days, a follow-up echocardiogram was performed on 12 patients, and none had evidence of valve dysfunction, or valve or ventricular, papillary muscle, or chordate injury.

\section{Efficacy Endpoint}

The device was successfully inserted in 16 patients $(100 \%)$. Hemodynamic indexes improved immediately once support was initiated. On average, the cardiac index and mean arterial pressure increased from $1.6 \pm 0.4$ 


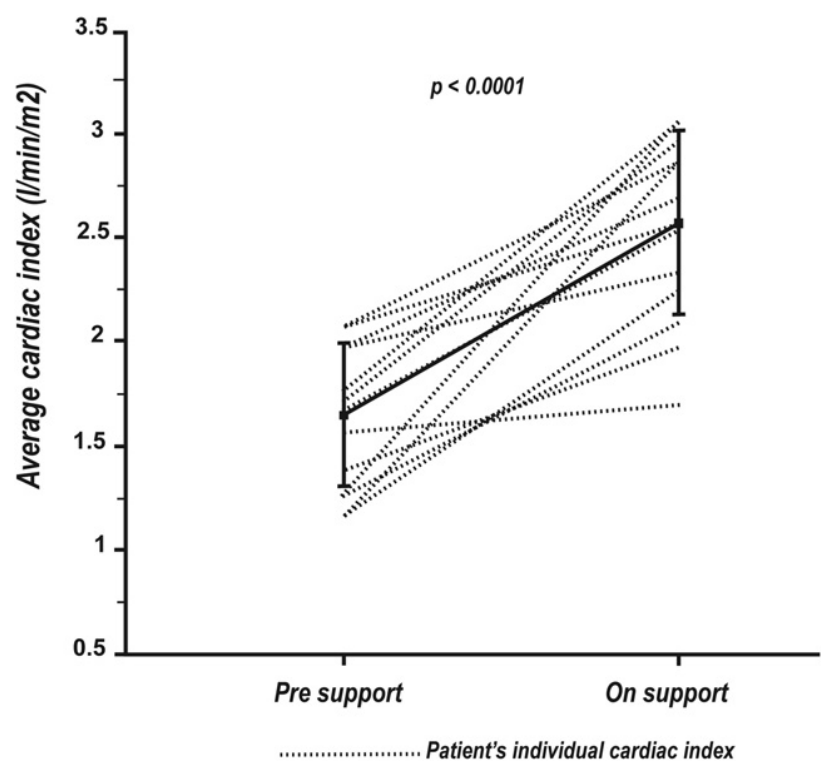

FIGURE 2. Average cardiac index and patient's individual cardiac index on support values reflect the average cardiac index measurements obtained within the first 24 hours after device implantation.

$\mathrm{L} / \mathrm{min} / \mathrm{m}^{2}$ to $2.5 \pm 0.4 \mathrm{~L} / \mathrm{min} / \mathrm{m}^{2}(P=.0001)$ and from 71.4 $\pm 12.5 \mathrm{~mm} \mathrm{Hg}$ to $83.1 \pm 7.5 \mathrm{~mm} \mathrm{Hg}(P=.01)$, respectively. Unloading was shown in a reduction of the pulmonary artery diastolic pressure from $28.0 \pm 3.9 \mathrm{~mm} \mathrm{Hg}$ to $19.8 \pm$ $3.2 \mathrm{~mm} \mathrm{Hg}(P<.0001)$. The patients' hemodynamics improved in conjunction with a decreased need for inotropic medications (Figures 2, 3, and 4).

Survival at 30 days, 6 months, and 1 year was $94 \%, 81 \%$, and $75 \%$, respectively. Of the 15 patients who survived to 30 days, 1 was successfully weaned off the Impella 5.0; he withdrew consent the next day and was still alive at the end of the study. Of the remaining patients, 13 (93\%) recovered their native heart function and $1(7 \%)$ did not show any evidence of ventricular recovery after 12.6 days of support. On removal of the Impella LD, this patient underwent implantation of an intracorporeal longer term LVAD (Jarvik 2000; Jarvik Heart, New York, NY) as a bridge to transplantation.

\section{DISCUSSION}

Approximately $0.2 \%$ to $6 \%$ of patients undergoing coronary or valvular cardiac procedures will develop PCCS. ${ }^{8}$ Increased inotropic and IABP support has been the most widely used initial strategy to support these patients. However, a major drawback to this treatment approach resides in the increased mortality associated with administration of inotropes, ${ }^{9}$ and the improvement of hemodynamic parameters and cardiac output ${ }^{10}$ is at the expense of increased myocyte death and derangement in the functionality of the remaining myocytes. ${ }^{11}$ In addition, the absence of active left ventricular unloading and the requirement for a certain residual level of left ventricular function with IABP might

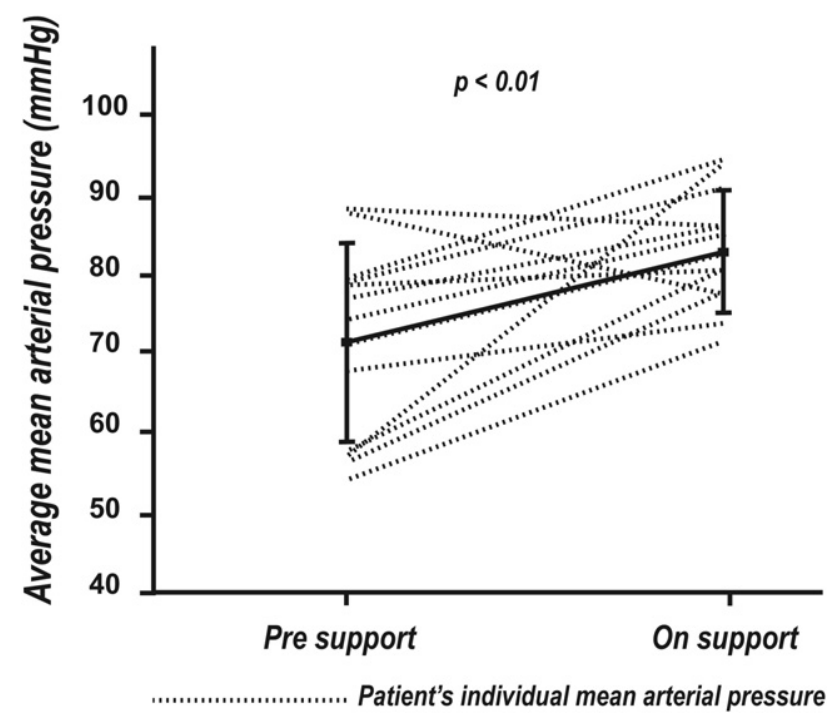

FIGURE 3. Average mean arterial pressure (MAP) and patient's individual MAP on support values reflect the average MAP measurements obtained within the first 6 hours after device implantation.

limit the chance for cardiac recovery and potentially increase the incidence of severe heart failure for the survivors.

For those patients refractory to inotropes and IABP support, VADs have been used as a last resort to save patients' lives. Overall, the results in patients with PCCS have been historically disappointing when heart recovery was sought. ${ }^{12-14}$ More recently, early implantation of complete mechanical circulatory assistance in the setting of PCCS has improved survival. In a number of reports, ${ }^{15-18}$ an improved outcome was obtained by active unloading of the ventricle and rapid restoration of normal end-organ perfusion. Thus, we approached our patients with the assumption that the early initiation of a minimally invasive potent mechanical circulatory support device with direct unloading capabilities would improve the outcomes compared with those of a more conservative strategy of escalation in vasoactive and inotropic medication, which has not proved to improve the outcomes in these settings. ${ }^{5,10}$

The protocol was designed under Food and Drug Administration guidance and allowed enrollment of a maximum of 20 patients in the study. Recruiting these sick patients has proved difficult and very challenging. The survival outcomes were known because the data could not be blinded in this open-label, single-arm trial. The study steering committee believed that enough clinical evidence was collected with 16 patients to document the safety and feasibility of the technology in consideration of the very favorable survival outcomes. The consensus was that the additional time (approximately 8 months) required to enroll additional patients was significant and that the information that could have been collected from the remaining few patients could not have changed the overall favorable safety trends 


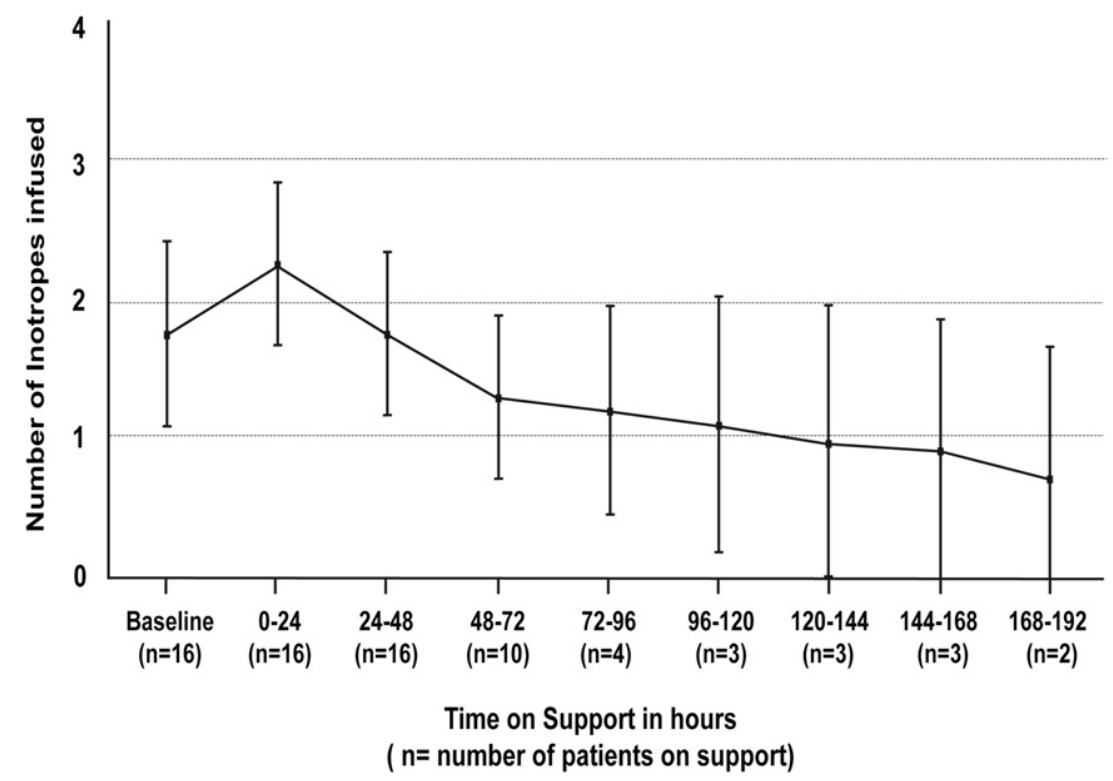

FIGURE 4. Average daily inotrope use.

significantly. Thus, in consultation with the Food and Drug Administration and the study sponsor, the steering committee decided to end the study and submit the results.

The results of our study have demonstrated that the use of the Impella enabled immediate restoration of hemodynamics with a gradual reduction in the need of inotropic support. Overall, $94 \%$ of patients survived to 30 days and, of those, $93 \%$ were weaned off mechanical support. The survival outcomes were favorable in our study compared with those for other VAD cohorts in postcardiotomy settings. ${ }^{19,20}$ Perhaps the early restoration of the hemodynamics prevented the installation of irreversible end organ dysfunction, which led to greater survival. In the present study, Impella support was initiated promptly when the patient experienced hemodynamic compromise despite inotropic support. In routine practice, patients experiencing PCCS or low cardiac output syndrome would most likely receive an increased dose of inotropes and an IABP before potent circulatory support with an invasive VAD is considered. We were anxious to evaluate this attractive one-of-a-kind, near-full-flow, yet catheterdelivered, LVAD in a postcardiotomy setting. The rationale for using the Impella short-term assist device as a "bridgeto-decision" or "bridge-to-recovery" lies in the need for a potent circulatory support system with low morbidity that can be implanted quickly and managed easily in emergent situations with catastrophic hemodynamic deterioration. It was also based on the belief that early implantation of the pump was appropriate for this patient population as confirmed by the poor patient hemodynamics before pump insertion.

The minimum amount of inotropic support required by the protocol to qualify for the study might have been conservative. However, all our patients required a higher dose than the protocol prespecified threshold of inotropic support before enrollment with a mean cardiac index of $1.6 \pm 0.4 \mathrm{~L} / \mathrm{min} / \mathrm{m}^{2}$. At inclusion, the patients were receiving an average of 2 high doses of inotropes/pressors for at least 15 minutes (dopamine or equivalent) to 120 minutes (milrinone) without improvement of their hemodynamics. The dose of inotropes (Figure 4) and pressors had to be increased shortly afterward to an average of 3 inotropes/ pressors, given the continuous deterioration of the hemodynamic status. After pump implantation, the hemodynamics improved significantly, and the inotropes were weaned off over time as the patient's condition improved. Moreover, the study investigators were asked to specify how they would have treated the patients they enrolled if the Impella were not available and the patient were to be treated outside of the study. To this question and, in the investigators' opinion, $14(88 \%)$ would have required a more invasive surgical LVAD and $2(12 \%)$ would have been treated with an IABP with a high dose of 3 inotropes or more. Finally, they would have considered delaying the escalation to a surgical LVAD, given the additional morbidity ensued from the implantation procedure.

In the present study, bleeding events were within the range of those reported in similar postcardiotomy studies, ${ }^{21}$ even though factors inherent to patient presentation and cardiac surgery with CPB predisposed these patients to an increased risk of microvascular bleeding requiring blood transfusion and/or re-exploration surgery. ${ }^{22}$ No bleeding events were related to the device itself. The sepsis rate in our study was comparable to that observed in studies of patients in $\mathrm{PCCS}^{23}$ or CS requiring hemodynamic support. ${ }^{24}$ All sepsis events resolved with antibiotic treatment. 
Detailed echocardiographic evaluation, performed by an independent core laboratory, did not show any evidence of structural damage to the heart during device use or in subsequent follow-up. The aortic valve remained intact without visible damage at all points, and there was no evidence of left ventricular or proximal aorta structural damage. These results are consistent with those of previous reports. ${ }^{16}$

The 2 device failures encountered during our study were most likely due to a kink of the driveline that resulted in blood entering the motor housing and leading to increased resistance and, ultimately, pump failure. The manufacturer has since released a kink-resistant catheter for the Impella 5.0/LD

The Impella 5.0/LD is a versatile, effective, low-cost, and significantly less-invasive LVAD able to directly unload the $\mathrm{LV}$ and provide up to $5 \mathrm{~L} / \mathrm{min}$ of flow to the general circulation. Because implantation of the pump only requires a low anticoagulation regimen, it is a very compelling alternative to surgical short-term VADs in the acute setting. These advantages might lower the decision threshold for the initiation of mechanical circulatory support to restore the hemodynamics and prevent the aggravation of endorgan dysfunction and potentially lead to improved outcomes. For patients who do not recover their native heart function, the device might provide time to adequately evaluate patients for potential long-term implantation of a durable VAD with a cost-effective bridge to transplant or destination therapy strategy.

The protocol was designed so that a surgeon could place the pump by way of the femoral artery and thus permanently close the sternal wound. Alternatively, the device could be placed directly by way of the ascending aorta, which required skin-only closure of the chest wound. In time, because of the expediency of avoiding femoral artery exposure, the ease of echocardiographic-guided placement, and concern about the potential for vascular injury $(21 \mathrm{~F}$ pump) or potential ischemia, the surgeons came to prefer the direct aortic approach.

In recent years, as the experience matured and the surgeons gained more confidence in implanting these catheter-based pumps, the peripheral approach has become the predominant implantation technique. The axillary approach appears to be preferable to the femoral approach, because it allows for early bed mobilization of the patient. ${ }^{17}$

\section{Study Limitations}

The major limitations of the present prospective, safety and feasibility study were the lack of a control group and the size and heterogeneity of the cohort enrolled, which did not allow an easy statistical comparison of our results with the results from other PCCS studies. Moreover, the extrapolation of our results to a general postcardiotomy patient population is limited, because, in some cases, cardiogenic shock occurred immediately after weaning from CPB and required rapid device implantation but in a few other cases, it occurred several hours to up to 24 hours after the patients were weaned off CPB.

\section{CONCLUSIONS}

The results from our study have shown that the use of the Impella 5.0/LD system is safe and feasible and provides excellent hemodynamic support in the settings of PCCS or low cardiac output syndrome. Compared with other surgical short-term LVADs, ${ }^{25}$ the less-invasive implantation, ease of use, lower cost, and favorable safety profile offered by this technology make it a very compelling and convenient alternative for both patients and surgeons.

We are grateful to the study investigators for their thoughtful feedback during the preparation of this report and above all to the patients who participated in our study to make this report possible.

\section{References}

1. Arafa OE, Pedersen TH, Svennevig JL, Posse E, Gerian O. Intraaortic balloon pump in open heart operations: 10-year follow-up with risk analysis. Ann Thorac Surg. 1998;65:741-7.

2. Baldwin RT, Slogoff S, Noon GP, Sekela M, Frazier OH, Edelman SK, et al. A model to predict survival at time of postcardiotomy intraaortic balloon pump insertion. Ann Thorac Surg. 1993;55:908-13.

3. DeRose JJ Jr, Umana JP, Argenziano M, Catanese KA, Levin HR, Sun BC, et al Improved results for postcardiotomy cardiogenic shock with the use of implantable left ventricular assist devices. Ann Thorac Surg. 1997;64:1757-62.

4. Hausmann H, Potapov EV, Koster A, Krabatsch T, Stein J, Yeter R, et al. Prognosis after the implantation of an intra-aortic balloon pump in cardiac surgery calculated with a new score. Circulation. 2002;106(12 Suppl 1):I203-6.

5. Samuels LE, Kaufman MS, Thomas MP, Holmes EC, Brockman SK, Wechsler AS. Pharmacological criteria for ventricular assist device insertion following postcardiotomy shock: experience with the Abiomed BVS system. J Card Surg. 1999;14:288-93

6. Garatti A, Colombo T, Russo C, Lanfranconi M, Milazzo F, Catena E, et al. Different applications for left ventricular mechanical support with the Impella Recover 100 microaxial blood pump. J Heart Lung Transplant. 2005;24:481-5.

7. Siegenthaler MP, Brehm K, Strecker T, Hanke T, Nötzold A, Olschewski M, et al. The Impella Recover microaxial left ventricular assist device reduces mortality for postcardiotomy failure: a three-center experience. J Thorac Cardiovasc Surg. 2004;127:812-22.

8. Sylvin EA, Stern DR, Goldstein DJ. Mechanical support for postcardiotomy cardiogenic shock: has progress been made? J Card Surg. 2010;25:442-54.

9. Cuffe MS, Califf RM, Adams KF Jr, Benza R, Bourge R, Colucci WS, et al. Short-term intravenous milrinone for acute exacerbation of chronic heart failure: a randomized controlled trial. JAMA. 2002;287:1541-7.

10. Fonarow GC. Pharmacologic therapies for acutely decompensated heart failure. Rec Cardiovasc Med. 2002;3(Suppl 4):S18-27.

11. Feldman DS, Elton TS, Sun B, Martin MM, Ziolo MT. Mechanisms of disease: detrimental adrenergic signaling in acute decompensated heart failure. Nat Clin Pract Cardiovasc Med. 2008;5:208-18.

12. Cabuay B, Hryniewicz K, Sun B, Feldman D. Door-to-VAD time: an expedient management strategy for cardiogenic shock. Curr Opin Cardiol. 2010;25 249-52.

13. Aggarwal S, Cheema F, Oz MC, Naka Y. Long-term mechanical circulatory support. In: Cohn III LH, ed. Cardiac surgery in the adult. New York, NY: McGrawHill; 2008. p. 1609-28

14. Meyns B, Sergeant P, Wouters P, Casselman F, Herijegers P, Daenen W, et al. Mechanical support with microaxial blood pumps for postcardiotomy left ventricular failure: can outcome be predicted? J Thorac Cardiovasc Surg. 2000;120 392-400.

15. Hernandez AF, Grab JD, Gammie JS. A decade of short-term outcomes in postcardiac surgery ventricular assist device implantation: data from the Society of Thoracic Surgeon's National Cardiac Database. Circulation. 2007;116:606-12. 
16. Meyns B, Dens J, Segeant P, Herijgers P, Daenen W, Flameng W. Initial experience with the Impella device in patients with cardiogenic shock-Impella support for cardiogenic shock. Thorac Cardiovasc Surg. 2003;51:312-7.

17. Sassard T, Scalabre A, Bonnefoy E, Sanchez I, Farhat F, Jegaden O. The right axillary approach for the Impella Recover LP 5.0 microaxial pump. Ann Thorac Surg. 2008;85:1468-70.

18. Garatti A, Colombo T, Russo C, Lanfranconi M, Milazzo F, Catena E, et al. Left ventricular mechanical support with the Impella Recover left direct microaxial blood pump: a single-center experience. Artif Organs. 2006;30:523-8.

19. John R, Long JW, Massey HT, Griffith BP, Sun BC, Tector AJ, et al. Outcomes of a multicenter trial of the Levitronix CentriMag ventricular assist system for shortterm circulatory support. J Thorac Cardiovasc Surg. 2011;141:932-9.

20. Rastan AJ, Dege A, Mohr M, Doll N, Flak V, Walther T, et al. Early and late outcomes of 517 consecutive adult patients treated with extracorporeal membrane oxygenation for refractory postcardiotomy cardiogenic shock. J Thorac Cardiovasc Surg. 2010;139:302e11, 311.e1.
21. De Robertis F, Birks EJ, Rogers P, Dreyfusa G, Pepper JR, Khaghania A. Clinical performance with the Levitronix Centrimag short-term ventricular assist device. J Heart Lung Transplant. 2006;25:181-6.

22. Despotis G, Avidan M, Eby C. Prediction and management of bleeding in cardiac surgery. J Thromb Haemost. 2009;7:111-7.

23. Körfer R, el-Banayosy A, Posival H, Minami K, Kizner L, Arusoglu L, et al. Mechanical circulatory support with the Thoratec assist device in patients with postcardiotomy cardiogenic shock. Ann Thorac Surg. 1996;61:314-6.

24. Cheng JM, den Uil CA, Hoeks SE, van der Ent M, Jewbali LSD, van Domburg RT, et al. Percutaneous left ventricular assist devices vs. intra-aortic balloon pump counterpulsation for treatment of cardiogenic shock: a metaanalysis of controlled trials. Eur Heart J. 2009;30:2102-8.

25. Lamarche Y, Cheung A, Ignaszewski A, Higgins J, Kaan A, Griesdale DEG, et al. Comparative outcomes in cardiogenic shock patients managed with Impella microaxial pump or extracorporeal life support. J Thorac Cardiovasc Surg. 2011; 142:60-5. 